\section{Signal Intensity of High B-value Diffusion-weighted Imaging for the Detection of Prostate Cancer}

\author{
Rezaeian A. ${ }^{* * \odot}$, Tahmasebi Birgani M. J. ${ }^{1}$, Chegeni N. ${ }^{1}$, \\ Sarkarian M. ${ }^{2}$, Hanafi M. Gh. ${ }^{3}$, Akbarizadeh Gh. ${ }^{4}$
}

\begin{abstract}
Background: Diffusion-weighted imaging (DWI) is a main component of multiparametric MRI for prostate cancer detection. Recently, high $b$ value DWI has gained more attention because of its capability for tumor characterization.

Objective: To assess based on histopathological findings of transrectal ultrasound (TRUS)-guided prostate biopsy as a reference, an increase in signal intensity of prostatic lesions in comparison with normal background tissue on high b-value diffusion-weighted images could be a sign of malignancy.

Material and Methods: Fifty-three consecutive patients retrospectively included in the study. All patients underwent routine TRUS-guided prostate biopsies involving 12 cores after the magnetic resonance imaging (MRI) examinations. In seventeen patients ( $\mathrm{n}=35$ lesions), the prostate cancer was histologically confirmed by TRUS-guided prostate biopsy. The biopsy results of other patients were negative. Signal intensities on the high b-value $\left(1600 \mathrm{~s} / \mathrm{mm}^{2}\right)$ images of the peripheral zone, the central gland, and the defined lesions were evaluated using region of interestbased measurements. Sensitivity, specificity, positive predictive value (PPV) and negative predictive value (NPV) for prostate cancer detection using signal intensity of high $b$ value diffusion-weighted images were calculated.
\end{abstract}

Results: In the patients with confirmed prostate cancer, fourteen had visually increased SI on the high b-value images. The SI of lesions for these patients was higher than the SI of peripheral zone $(22 \pm 18 \%)$ or central gland $(31 \pm 20 \%)$. In patients with a negative biopsy, eight had visually increased SI on the high b-value images. The SI of lesions for these patients was $23 \pm 21 \%$ and $35 \pm 18 \%$ higher than the SI in the peripheral zone and the central gland, respectively. The sensitivity, specificity, PPV, and NPV for prostate cancer using SI of high b value DWI were 71, 87, 62, and 87 $\%$, respectively.

Conclusion: Visually increased SI on the high b-value images can be an indication of malignancy, although some benign lesions also show this increase in signal intensity.

Citation: Rezaeian A, Tahmasebi Birgani M. J, Chegeni N, Sarkarian M, Hanafi M. Gh, Akbarizadeh Gh. Signal Intensity of High B-value Diffusion-weighted Imaging for the Detection of Prostate Cancer. J Biomed Phys Eng. 2019;9(4):453-458. https://doi.org/10.31661/jbpe.v0i0.811.

\section{Keywords}

Diffusion Magnetic Resonance Imaging • Prostatic Neoplasms • Predictive Value of Tests

\section{Introduction}

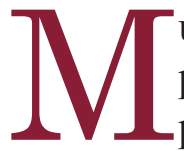

ultiparametric magnetic resonance imaging (mpMRI) of the prostate is a powerful tool for detection and localization of prostate cancer that combines conventional morphological T2-weighted imaging with various functional MR techniques such as
${ }^{1}$ Department of Medical Physics, Faculty of Medicine, Ahvaz Jundishapur University of Medical

Sciences, Ahvaz, Iran

${ }^{2}$ Department of Urology,

Golestan Hospital, Ahvaz

Jundishapur University

of Medical Sciences,

Ahvaz, Iran

${ }^{3}$ Department of Radiol-

ogy, Faculty of Medicine,

Ahvaz Jundishapur

University of Medical Sci-

ences, Ahvaz, Iran

${ }^{4}$ Department of Electri-

cal Engineering, Faculty

of Engineering, Shahid

Chamran University of

Ahvaz, Ahvaz, Iran

*Corresponding author:

A. Rezaeian

Department of Medical

Physics, Faculty of Medi-

cine, Ahvaz Jundishapur

University of Medical

Sciences, Ahvaz, Iran

E-mail: abbas.rezaian@ yahoo.com

Received: 2 August 2017 Accepted: 2 September 2017 
dynamic contrast-enhanced MRI, diffusionweighted imaging (DWI), and spectroscopy. DWI is less-time consuming compared to other functional techniques and is performed without contrast media [1]. The contrast in the diffusion-weighted images is determined by differences in motion of water molecules in different tissues that this mobility of water molecules is affected by histological features such as tissue cellularity [2]. DWI can evaluate differences in water molecule diffusion by qualitative visual assessment using relative signal intensity (SI) and also by quantitative assessment calculating the apparent diffusion coefficient (ADC) from diffusion-weighted images [3]. Malignant changes of the prostate gland that are often associated with increased cell density and loss of lumenal and ductal spaces, leading to more restrictions for diffusion of water molecules, can be effectively probed by diffusion-weighted imaging $[4,5]$. In DWI the hypointensity on the ADC map is an indication of prostate cancer [6, 7]. It has recently been reported that DW images obtained by ultrahigh $b$-values provide a good contrast between cancerous and background tissue [811]. The European Society of Urogenital Radiology (ESUR) published guidelines for the standardization, evaluation, and reporting of prostate MRI that include a scoring system named PI-RADS (Prostate Imaging Reporting and Data System) in which abnormalities in the prostate received a score ranging from 1 to 5 [12]. PI-RADS Assessment of DWI includes an ADC map and high b-value images. A lesion with an increased SI on the high $b$ value diffusion-weighted images and decreased signal intensity on the ADC map is given 5 out of 5 possible scores in the PI-RADS scoring system. The reduced signal on the ADC map of suspect lesions is routinely applied in the clinical practice. However, the effect of high and ultra-high b-values on the visualization of prostate cancer using native DW images remains unclear. Therefore, this study aims to investigate the increase in SI of the high b-val- ue diffusion-weighted images of prostate suspecting lesions with the aid of histopathological findings for TRUS-guided prostate biopsy as a reference.

\section{Material and Methods}

\section{Patients}

This retrospective single-institution study was approved by the institutional review board and written informed consent was obtained from every patient. From April 2016 to April 2017, 53 consecutive patients with clinically suspected prostate cancer, such as positive findings on digital rectal examination or elevated serum prostate-specific antigen (PSA) level ( $\geq 4 \mathrm{ng} / \mathrm{mL}$ ), were enrolled in our study. All patients underwent routine TRUS-guided prostate biopsies involving 12 cores after the MRI examination. No MRI-targeted biopsies were performed. Patients with prior hormonal, surgical, or irradiation therapies were excluded and median PSA level also was $9.49 \mathrm{ng} / \mathrm{mL}$ (range, 3.2-156 ng/mL).

\section{MRI Data Acquisition}

MRI examinations were performed on a 1.5T whole-body MRI scanner (Magnetom Essenza, Siemens Medical Systems, Erlangen, Germany). Patients were imaged in the supine position using a 6-channel pelvic phased-array coil then an endorectal coil was not employed. All patients underwent T2-weighted imaging (T2WI) and DWI and finally no bowel preparation was performed. Turbo spin-echo T2-weighted images (in-plane resolution $0.57 \times 0.57 \mathrm{~mm}^{2}$, repetition and echo time TR/ TE 3500-3900/100 msec, a slice thickness/interslice gap setting of $4 / 0.5 \mathrm{~mm}, 20$ sections, field of view $280 \times 280 \mathrm{~mm}^{2}$, flip angle $150^{\circ}$ ) covering the whole prostate and seminal vesicles were acquired in axial and sagittal planes.

Diffusion-weighted imaging was performed using a single-shot echo planar imaging sequence in the transverse plane during free breathing with the following parameters: rep- 
etition and echo time TR/TE 4200/110 msec; a slice thickness/interslice gap setting of $4 / 0.5$ $\mathrm{mm}$; field of view $250 \times 250 \mathrm{~mm}^{2}$; number of scan average (NSA) 6; acquisition matrix $110 \times 110$; parallel imaging factor of 2 ; receiver bandwidth $1188 \mathrm{~Hz} /$ voxel; EPI factor 70; bvalues of 0,800 and $1600 \mathrm{~s} / \mathrm{mm}^{2}$.

\section{Measurement}

Images were analyzed by one experienced radiologist who was not involved in image acquisition and was blind to patient clinical information. First, an increase in SI of high bvalue images was assessed by the radiologist. The location of lesions on the high b-value $\left(1600 \mathrm{~s} / \mathrm{mm}^{2}\right)$ diffusion-weighted images were compared to the biopsy specimen locations. The reference standard was judged by systematic evaluation of biopsy results: regions in biopsy specimens showing pathological evidence of cancer were considered as positive, whereas regions showing non-presence of cancer were considered as negative. Regions of interest (ROIs) were drawn upon the lesion, the peripheral zone and the central gland of the prostate. Signal intensities on the high b-value $\left(1600 \mathrm{~s} / \mathrm{mm}^{2}\right)$ images of the peripheral zone, the central gland, and the defined lesions were evaluated using ROI-based measurements and the picture archiving and communication system (PACS). The b-value images were normalized for each patient by dividing signal intensity of ROIs by the mean value of an ROI placed within the internal obturator muscle in the proximity of the prostate. Normalization of high b-value images compensates the bias resulting from variability between patients. Throughout the measurement, we used "copy" and "paste" of the ROI to standardize the ROI sizes and avoid sampling errors.

The statistical analysis was performed using IBM SPSS Statistics 19 for Windows (SPSS, Chicago, IL, USA). A P value $<0.05$ was considered significantly. A student-paired t-test was used to compare SI of malignant lesions and peripheral zone or central gland tissues.
Sensitivity, specificity, positive predictive value (PPV) and negative predictive value (NPV) for prostate cancer detection using SI of high $b$ value DWI were calculated. Lesions that showing pathological evidence of cancer in biopsy specimens and increased signal intensity in high $b$ value diffusion-weighted images were considered as true positive. Lesions showing no-presence of cancer in biopsy specimens and no increase in signal intensity were considered as true negative.

\section{Results}

In this study fifty three patients were involved. Seventeen patients had histologically confirmed prostate cancer in 35 lesions. Twenty-two of these tumors were located in the peripheral zone and thirteen of them in the central and transitional zone. Thirty-three patients had negative biopsies. In the patients with confirmed prostate cancer, fourteen had visually increased SI on the high b-value $\left(1600 \mathrm{~s} / \mathrm{mm}^{2}\right)$ images (Figure 1). The SI of lesions for these patients was higher than the SI of peripheral zone $(22 \pm 18 \%)$ or central gland $(31 \pm 20 \%)$.

Signal intensity on the high $b$ value diffusion-weighted images increased in eight patients with negative biopsies (Figure 2). The SI of these lesions was $23 \pm 21 \%$ and $35 \pm 18 \%$ higher than the SI in the peripheral zone and the central gland, respectively. The increase in $\mathrm{SI}$ in the cancerous and non-cancerous lesions in comparison with the peripheral zone and the central gland was highly significant $(\mathrm{P}<0.001$, respectively) than patients without visually increased SI on the high b-value images. Altogether, $71 \%$ of lesions with increased SI on the high b-value images were lesions with confirmed prostate cancer. Malignant lesions with increased SI were not visually distinguishable from non-tumor lesions with increased SI on the high b-value images.

The sensitivity, specificity, PPV, and NPV for prostate cancer detection using SI of high b value DWI were $75,82,66$, and $87 \%$, respectively. 


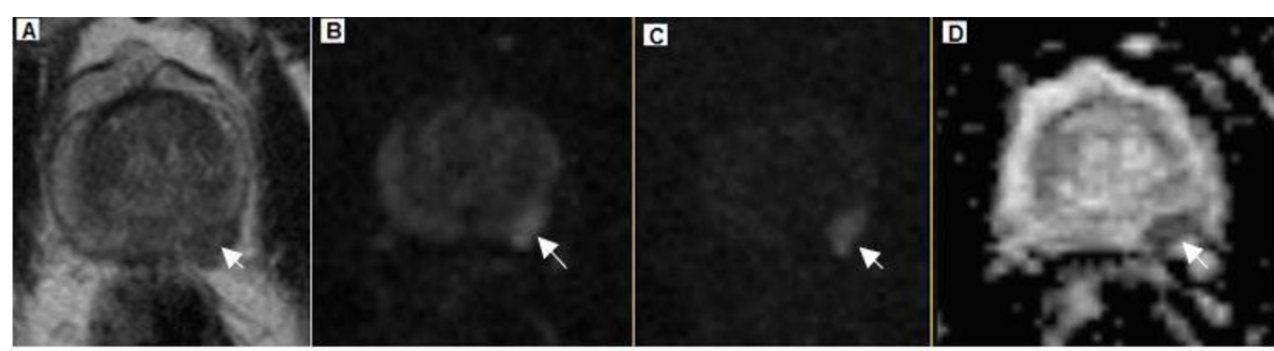

Figure 1: A 67-year-old patient with TRUS-guided biopsy-proven prostate cancer, Gleason Score $4+5=9$ and PSA level of $4.8 \mathrm{ng} / \mathrm{ml}$. (A) Transverse T2- weighted image shows a hypointense area (arrow) in the peripheral zone of the left lobe. (B) Transverse DW image $\left(b=800 \mathrm{~s} / \mathrm{mm}^{2}\right)$. (C) Transverse DW image $\left(b=1600 \mathrm{~s} / \mathrm{mm}^{2}\right.$ ) clearly demonstrates hyperintense area (arrow) in the peripheral zone of the left lobe. (D) Apparent diffusion coefficient (ADC) map with reduced signal intensity (arrow).

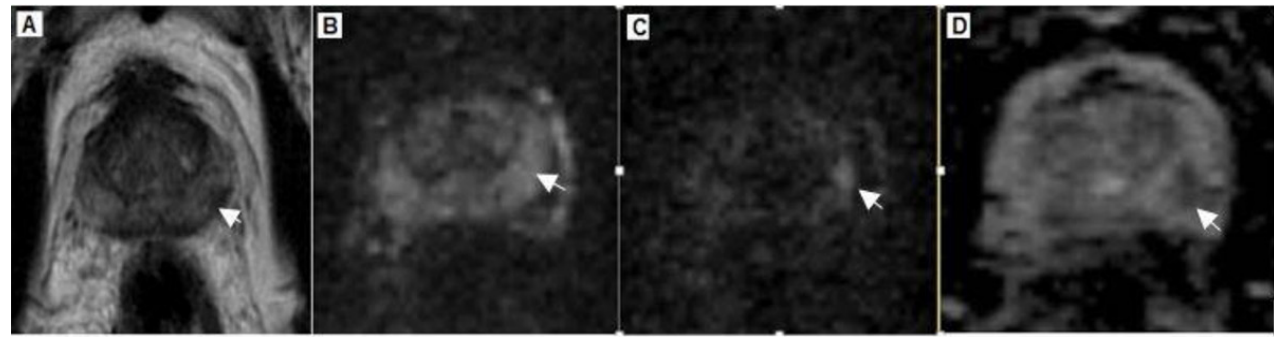

Figure 2: A 67-year-old patient with increased $\mathrm{SI}$ on the high b-value images and a negative biopsy. (A) Transverse T2- weighted image shows a hypointense area (arrow) in the peripheral zone of the left lobe. (B) Transverse DW image $\left(b=800 \mathrm{~s} / \mathrm{mm}^{2}\right)$. (C) Transverse DW image $\left(b=1600 \mathrm{~s} / \mathrm{mm}^{2}\right)$ clearly demonstrates hyperintense area (arrow) in the peripheral zone of the left lobe. (D) Apparent diffusion coefficient (ADC) map with reduced signal intensity (arrow).

\section{Discussion}

DWI, as a part of multiparametric MR approach, is a promising method for detection and characterization of prostate cancer. DWI probes the mobility of water molecules in tissue at a micrometer scale. Diffusion MR imaging can evaluate differences in motion of water molecular by qualitative visual assessment using relative signal intensity and also by quantitative assessment calculating the ADC [3]. ADC values in prostate cancer are considerably lower than those of normal prostate tissue $[13,14]$. In prostate cancer, the loss of large lumenal and ductal spaces of normal glandular tissue and an increase in cell density lead to decreasing diffusivity of water molecules [15]. In addition to ADC, the signal intensity of high-b-value DWI has recently gained much attention and shown a capability for tissue and tumor characterization and detection $[11,16]$. The recently released guidelines for prostate MRI from the ESUR recommends the inclusion of high- $b$ value images $(b$ values of $1400-2000 \mathrm{sec} / \mathrm{mm}^{2}$ ) to the recommended sequences obtained during mpMRI of the prostate. On the high-b value images, the signal intensity of the normal prostate tissue background is low, whereas clinically significant cancers demonstrate high signal intensity. The diffusion $b$ value (unit, $\mathrm{s} / \mathrm{mm}^{2}$ ) controls the degree of diffusion weighting within the DWI pulse sequence. 
In this study, there were lesions with visually increased SI on the high b-value images that their SI was significantly higher than the SI of surrounding tissue. About $71 \%$ of these lesions were tumors. However, $29 \%$ of lesions with increased SI were histologically noncancerous after systematic TRUS-guided prostate biopsy. Nevertheless, visually increased SI on the high b-value images is a sign of malignancy.

The $b$ value used in DWI strongly influences the signal intensity of the lesions on the high $b$ value images. However, the reason for the increase in SI on the high $b$ value images of some cancerous or even benign lesions is not exactly clear. Cellular tissues with highly restricted water regions generally demonstrate high signal intensity on high- $b$ value images. However, in some cases, the increased signal intensity on the diffusion-weighted images cannot be attributed to limitations in water mobility, but they arise from tissues with intrinsically long T2-relaxation times; an effect is known as "T2-shine through". This effect could be overcome by the use of $b$ values greater than $1000 \mathrm{~s} / \mathrm{mm}^{2}$. Given that in this study DW images were obtained by b-value of $1600 \mathrm{~s} / \mathrm{mm}^{2}$, the impact of the T2 effect might be limited.

The sensitivity, specificity, PPV, and NPV for prostate cancer detection using SI of high b value DWI were 75, 82, 66, and $87 \%$, respectively. Although the sensitivity and PPV of this study were relatively less than some other studies, however, due to the differences in equipment and method used for correlation of radiologic with pathologic findings, it is difficult to compare the accuracy of various studies. Several possible etiologies can be suggested for the low accuracies in our study. First, imaging was performed using a torso phased-array coil; use of an endorectal coil would have resulted in increased SNR and been expected to yield higher accuracy for tumor localization. Second, we assessed high b value diffusion-weighted images, but not the corresponding ADC map. Finally, the standard of reference for comparing SI of background normal tissue and prostate cancer was TRUS guided prostate biopsies instead of prostatectomy specimens. It should be noted that positive results of even repeated TRUS-guided biopsies in patients with elevated PSA are not always true. The results of our study could be affected by this issue that tend to underestimate the calculated sensitivity and PPV.

Additional limitations of our study include the following. First, the systematic biopsy was used for determination of lesions. Considering that lesion- guided biopsies were not conducted, there was not the exact correspondence between MRI finding and biopsy results. Second, our study was limited by a relatively small sample size. Third, in our study, the suspected prostate cancer patients were examined using a 1.5 T MRI system with 8- channel external coil. Because of the higher SNR and improved spatial resolution at $3 \mathrm{~T}, 3 \mathrm{~T}$ MRI system can improve the diagnostic accuracy for detection of prostate cancer.

\section{Conclusion}

In conclusion, the findings in this study suggest that the high b-value DWI can improve diffusion contrast between prostate cancer lesions and normal background tissue. Moreover, increased signal intensity on the high bvalue diffusion-weighted images can be a useful sign of malignancy.

\section{Acknowledgment}

This work is a part of the Ph.D. thesis which is financially supported by Ahvaz Jundishapur university of medical sciences (Grant No. 94186).

\section{Conflict of Interest}

None

\section{References}

1. Zhang K, Shen Y, Zhang X, Ma L, Wang H, An $\mathrm{N}$, et al. Predicting Prostate Biopsy Outcomes: A Preliminary Investigation on Screening with 
Ultrahigh B-Value Diffusion-Weighted Imaging as an Innovative Diagnostic Biomarker. PLOS One. 2016;11:e0151176. doi: 10.1371/journal.pone.0151176. PubMed PMID: 26963936; PubMed Central PMCID: PMC4786278.

2. Kim TH, Jeong JY, Lee SW, Kim CK, Park BK, Sung $\mathrm{HH}$, et al. Diffusion-weighted magnetic resonance imaging for prediction of insignificant prostate cancer in potential candidates for active surveillance. Eur Radiol. 2015;25:1786-92. doi: 10.1007/ s00330-014-3566-2. PubMed PMID: 25636416.

3. Le Bihan D. Apparent diffusion coefficient and beyond: what diffusion MR imaging can tell us about tissue structure. Radiology. 2013;268:31822. doi: 10.1148/radiol.13130420. PubMed PMID: 23882093.

4. Maas MC, Futterer JJ, Scheenen TW. Quantitative evaluation of computed high $B$ value diffusion-weighted magnetic resonance imaging of the prostate. Invest Radiol. 2013;48:779-86. doi: 10.1097/RLI.0b013e31829705bb. PubMed PMID: 23907102.

5. Chatterjee A, Watson G, Myint E, Sved P, McEntee M, Bourne R. Changes in Epithelium, Stroma, and Lumen Space Correlate More Strongly with Gleason Pattern and Are Stronger Predictors of Prostate ADC Changes than Cellularity Metrics. Radiology. 2015;277:751-62. doi: 10.1148/radiol.2015142414. PubMed PMID: 26110669.

6. Mazaheri Y, Shukla-Dave A, Hricak H, Fine SW, Zhang J, Inurrigarro G, et al. Prostate cancer: identification with combined diffusion-weighted MR imaging and 3D 1H MR spectroscopic imaging-correlation with pathologic findings. Radiology. 2008;246:480-8. doi: 10.1148/radiol.2462070368. PubMed PMID: 18227542

7. Hambrock T, Somford DM, Huisman HJ, Van Oort IM, Witjes JA, Hulsbergen-van de Kaa CA, et al. Relationship between apparent diffusion coefficients at 3.0-T MR imaging and Gleason grade in peripheral zone prostate cancer. Radiology. 2011;259:453-61. doi: 10.1148/radiol.11091409. PubMed PMID: 21502392.

8. Wetter A, Nensa F, Lipponer C, Guberina N, Olbricht T, Schenck M, et al. High and ultra-high b-value diffusion-weighted imaging in prostate cancer: a quantitative analysis. Acta Radiol. 2015;56:100915. doi: $10.1177 / 0284185114547900$. PubMed PMID: 25168023.
9. Ueno Y, Kitajima K, Sugimura K, Kawakami F, Miyake $\mathrm{H}$, Obara $\mathrm{M}$, et al. Ultra-high b-value diffusion-weighted MRI for the detection of prostate cancer with 3-T MRI. J Magn Reson Imaging. 2013;38:154-60. doi: 10.1002/jmri.23953. PubMed PMID: 23292979.

10. De Perrot T, Scheffler M, Boto J, Delattre BM, Combescure C, Pusztaszeri M, et al. Diffusion in prostate cancer detection on a $3 \mathrm{~T}$ scanner: How many b-values are needed? J Magn Reson Imaging. 2016;44:601-9. doi: 10.1002/jmri.25206. PubMed PMID: 26914964.

11. Agarwal HK, Mertan FV, Sankineni S, Bernardo M, Senegas J, Keupp J, et al. Optimal high b-value for diffusion weighted MRI in diagnosing high risk prostate cancers in the peripheral zone. J Magn Reson Imaging. 2017;45:125-31. doi: 10.1002/ jmri.25353. PubMed PMID: 27383502.

12. Weinreb JC, Barentsz JO, Choyke PL, Cornud F, Haider MA, Macura KJ, et al. PI-RADS Prostate Imaging - Reporting and Data System: 2015, Version 2. Eur Urol. 2016;69:16-40. doi: 10.1016/j. eururo.2015.08.052. PubMed PMID: 26427566.

13. Tamada T, Sone T, Jo Y, Toshimitsu S, Yamashita T, Yamamoto A, et al. Apparent diffusion coefficient values in peripheral and transition zones of the prostate: comparison between normal and malignant prostatic tissues and correlation with histologic grade. J Magn Reson Imaging. 2008;28:720-6. doi: 10.1002/jmri.21503. PubMed PMID: 18777532.

14. Renard Penna R, Cancel-Tassin G, Comperat E, Mozer P, Leon P, Varinot J, et al. Apparent diffusion coefficient value is a strong predictor of unsuspected aggressiveness of prostate cancer before radical prostatectomy. World J Urol. 2016;34:1389-95. doi: 10.1007/s00345-016-1789-3. PubMed PMID: 26902878.

15. Bourne R, Kurniawan N, Cowin G, Sved P, Watson G. $16 \mathrm{~T}$ diffusion microimaging of fixed prostate tissue: preliminary findings. Magn Reson Med. 2011;66:244-7. doi: 10.1002/mrm.22778. PubMed PMID: 21695726.

16. Grant KB, Agarwal HK, Shih JH, Bernardo M, Pang $\mathrm{Y}$, Daar D, et al. Comparison of calculated and acquired high $b$ value diffusion-weighted imaging in prostate cancer. Abdom Imaging. 2015;40:578-86. doi: 10.1007/s00261-014-0246-2. PubMed PMID: 25223523; PubMed Central PMCID: PMC5540661. 